\section{Disección submucosa endoscópica en cáncer gástrico incipiente: experiencia inicial en el Hospital Clínico de la Pontificia Universidad Católica de Chile}

\author{
ANDRÉS DONOSO D. ${ }^{1}$, ALLAN SHARP ${ }^{1}$, ADOLFO PARRA-BLANCO ${ }^{2}$, \\ JUAN CARLOS ROA ${ }^{3}$, JEAN PHILLIPE BÄCHLER ${ }^{\mathrm{a}}$, \\ FERNANDO CROVARI ${ }^{1}$, RICARDO FUNKE ${ }^{1}$, \\ FERNANDO PIMENTEL ${ }^{1}$, LUIS IBÁÑEZ ${ }^{1}$, SERGIO GUZMÁN ${ }^{1}$
}

\section{Endoscopic submucosal dissection in early gastric cancer. Experience in 16 patients}

Background: Endoscopic submucosal dissection (ESD) is a minimally invasive procedure that allows curative treatment of early gastric cancer (EGC) in selected patients. Aim: To report our initial experience with ESD. Material and Methods: Analysis of prospective data from 16 patients aged 61 to 84 years, who underwent ESD between December 2011 and June 2014. Tumor type, operative time, hospitalization length, oncologic outcomes, complications and short-term follow up were registered. Results: En-block resection was achieved in all cases. The median operative time was $135 \mathrm{~min}$ (range: 50-320 min). Specimens' median size was $3.5 \mathrm{~cm}$ (range: 3-10). All the resections were R0. In 14 patients ESD was considered curative. In two patients, ESD was considered potentially non-curative due to the presence pathological risk factors for lymph-node metastases in the biopsy specimen. Both patients underwent laparoscopic gastrectomy with lymph-node dissection. There was one case of gastric wall perforation that was repaired by laparoscopic suture. There was no mortality. The median follow-up time was 15 months (range: 2-30 months). Conclusions: ESD is a feasible and safe procedure in our institution with good results in this initial experience.

(Rev Med Chile 2015; 143: 1277-1285)

Key words: Endoscopy, digestive system; Early detection of cancer; Minimally invasive surgical procedures; Stomach neoplasms.

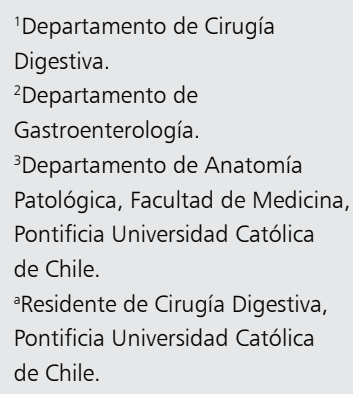

Recibido el 22 de octubre de 2014, aceptado el 13 de julio de 2015.

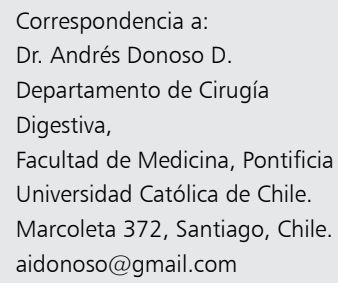

E 1 cáncer gástrico es la primera causa de muerte por cáncer en Chile $^{1}$ y es responsable de cerca de 3.000 fallecimientos al año ${ }^{2}$. Su pronóstico depende en gran medida de la etapa en la que se realiza el diagnóstico. En etapas precoces los pacientes tienen una sobrevida a 5 años cercana a $90 \%$, la que disminuye a $10 \%$ para pacientes con enfermedad metastásica ${ }^{3,4}$. La gastrectomía total o sub-total con disección linfática continúa siendo el pilar fundamental del tratamiento del cáncer gástrico, sin embargo, se asocia a morbilidad y mortalidad $^{5,6}$, y genera un impacto en la calidad de vida de los pacientes ${ }^{7,8}$.

Se define como cáncer gástrico incipiente (CGI) al adenocarcinoma limitado a la mucosa o submucosa del estómago independiente del compromiso linfático ${ }^{9}$. Este se caracteriza por su excelente pronóstico, con sobrevida a 10 años superior a $90 \%$ en pacientes sin metástasis linfáticas ${ }^{10-12}$. Hacia finales de la década de 1990, se desarrolló en Japón un nuevo tratamiento para los pacientes con CGI, la disección submucosa endoscópica o 
"ESD" (endoscopic submucosal dissection), procedimiento endoscópico que permite la resección completa y en bloque del tumor ${ }^{13}$, con resultados de sobrevida comparables a la cirugía, y con las ventajas de ser un procedimiento mínimamente invasivo que preserva el estómago ${ }^{14,15}$.

En el Hospital Clínico de la Pontificia Universidad Católica de Chile, la disección submucosa endoscópica (DSE) se realiza desde diciembre de 2011 en casos seleccionados de tumores precoces del tubo digestivo. El objetivo de este trabajo es dar a conocer nuestra experiencia inicial y los resultados inmediatos y a corto plazo de la DSE en CGI.

\section{Pacientes, Materiales y Métodos}

\section{Selección de pacientes}

Los pacientes son seleccionados de acuerdo a los criterios extendidos publicados por Gotoda y cols $^{16,17}$ (Tabla 1). Todo paciente con diagnóstico de CGI confirmado por biopsia endoscópica, es sometido a una endoscopia diagnóstica con luz blanca y cromoendoscopia con índigo carmín para determinar los límites de la lesión, el tamaño y la presencia o ausencia de ulceración. Se consideran candidatos a DSE pacientes con tumores bien diferenciados $<3 \mathrm{~cm}$ en caso de presentar ulceración o de mayor tamaño si es que no presentan esta característica y que clínicamente impresionen como intramucosos. También se incluyen pacientes con sospecha de invasión mínima de la submucosa (SM1) exclusivamente si la lesión es $<3 \mathrm{~cm}$, bien diferenciada y sin ulceración. Si bien, los criterios

\section{Tabla 1. Criterios extendidos de selección para DSE según Gotoda y cols.}

\section{CGI con muy bajo riesgo de metástasis linfáticas:}

- Adenocarcinoma bien diferenciado

o Clínicamente intramucoso:

- No ulcerado: sin límite de diámetro

- Ulcerado: $\leq 30 \mathrm{~mm}$ de diámetro

o Clínicamente SM1:

No ulcerado, $\leq 30 \mathrm{~mm}$ de diámetro

- Adenocarcinoma mal diferenciado*

o Clínicamente intramucoso:

No ulcerado, $\leq 20 \mathrm{~mm}$ de diámetro

*En nuestro grupo no incluimos en forma rutinaria estos casos para DSE y sólo se considera en casos de pacientes con riesgo quirúrgico muy elevado. extendidos también incluyen tumores poco diferenciados cuando hay compromiso exclusivo de la mucosa, la lesión es $<2 \mathrm{~cm}$ y no presenta ulceración, en nuestro grupo no consideramos en forma rutinaria estos casos para DSE. La evaluación de la invasión en profundidad de la pared se realizó con FICE (flexible spectral imaging color enhancement) + magnificación $^{18}$, NBI (narrow-band imaging) + foco cercano ${ }^{19}$ y/o endosonografía ${ }^{20}$ de acuerdo a cada caso. Todos los pacientes seleccionados son informados de las alternativas terapéuticas, incluyendo la gastrectomía (abierta o laparoscópica) y firman consentimiento informado previo al procedimiento.

\section{Preparación del procedimiento}

Los pacientes son hospitalizados el mismo día del procedimiento. En todos los casos la DSE se realizó en pabellón bajo anestesia general con el paciente en decúbito lateral izquierdo. La ubicación de los equipos y los distintos implementos se detalla en la Figura 1.

\section{Técnica de DSE}

La técnica de DSE ha sido descrita ampliamente ${ }^{21-24}$. Básicamente se tiñe el tumor con índigo carmín y se marca todo su perímetro en mucosa sana unos 3 a $5 \mathrm{~mm}$ fuera de la lesión. Posteriormente se eleva el tumor inyectando la submucosa con una solución de hialuronato de sodio a $0,4 \%$ + índigo carmín. Luego de elevar el tumor se corta la mucosa (por fuera de las marcas) para así acceder a la submucosa la que se diseca bajo visión directa hasta resecar completamente la lesión. Finalmente, una vez extraída, la pieza se estira con alfileres sobre una superficie plana y se envía en formalina a biopsia (Figura 2).

\section{Post-operatorio}

Los pacientes se mantienen en ayuno hasta el día siguiente en que comienzan dieta líquida. Durante la hospitalización reciben omeprazol por vía parenteral ( $40 \mathrm{mg}$ cada $6 \mathrm{~h}$ ). Los pacientes que evolucionan favorablemente son dados de alta al segundo día con indicación de dieta blanda y omeprazol por vía oral (40 mg cada $8 \mathrm{~h}$ ).

\section{Equipos e instrumental utilizados}

En todos los procedimientos se utilizó un endoscopio especialmente diseñado para DSE (EG450RD5; Fujifilm Corp., Tokyo, Japan) con un 

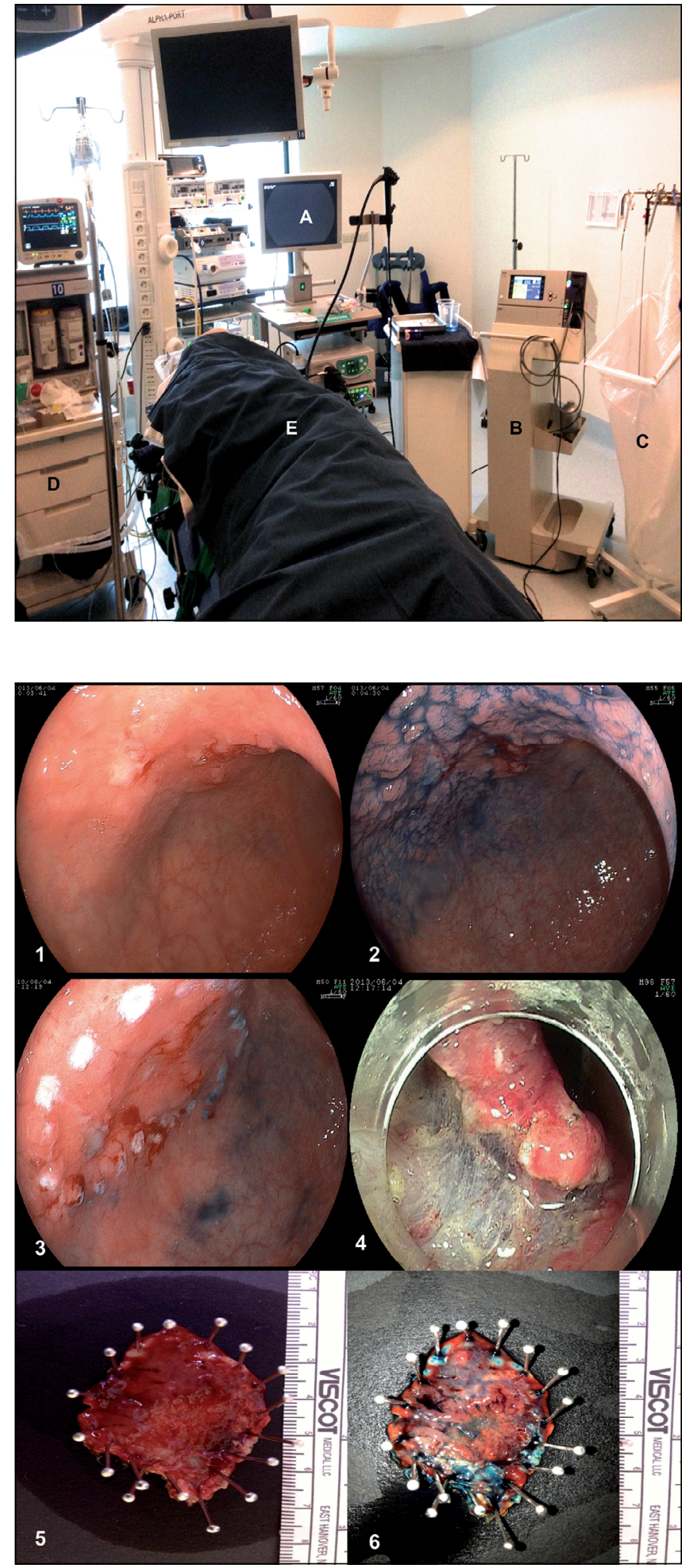

Figura 1. Ubicación de los equipos durante el procedimiento: (A) Torre de endoscopia; (B) Equipo de electrocirugía; (C) Atril para el instrumental endoscópico; (D) Máquina de anestesia; (E) Paciente.

Figura 2. Etapas de la DSE: (1) CGI ubicado en la pared anterior del cuerpo gástrico; (2) Tinción con índigo carmín; (3) Marcación de la lesión; (4) Disección de la submucosa; (5) Pieza resecada; (6) Pieza resecada teñida con índigo carmín. 
espaciador distal en su punta, que permite lograr una buena visualización y poder realizar maniobras de contra-tracción (D-201-11304; Olympus o DH-15GR; Fujifilm Corp). En 3 casos, se ocupó como método de tracción adicional un sistema de Clip-banda ${ }^{25}$. El bisturí endoscópico utilizado fue el Flush-Knife BT (DK-2623J, Fujifilm Optical Co, Ltd, Tokyo Japan). Como instrumentos auxiliares se utilizó el IT-Knife 2 (KD-611L, Olympus) y la pinza Coagrasper (FD-410 LR, Olympus). El equipo de electrocirugía utilizado fue el VIO 200 D (ERBE Elektromedizin $\mathrm{GmbH}$, Tübingen, Germany).

\section{Análisis histopatológico}

Todas las piezas resecadas fueron analizadas en su totalidad mediante mapeo con cortes seriados cada $2 \mathrm{~mm}^{16}$ de acuerdo al eje mayor del tumor informando los siguientes puntos:

- Diámetros mayor y menor de la pieza resecada.

- Diámetros mayor y menor del tumor.

- Tipo histológico y grado de diferenciación.

- Estado de los márgenes laterales y profundo.

- Presencia o ausencia de ulceración.

- Invasión linfovascular.

- Nivel máximo de invasión (adenocarcinoma in situ, intramucoso o submucoso).

- En caso de invasión de la submucosa, se informó el compromiso máximo medido en micras desde la muscular de la mucosa.

\section{Clasificación de las resecciones de acuerdo al análisis histopatológico}

En todos los casos, se determinaron los siguientes puntos ${ }^{26}$ :

1. Resección en bloque (extracción completa del tumor con márgenes macroscópicos libres y en una sola pieza).

2. Resección R0 (márgenes laterales y profundos sin tumor microscópico).

3. Ausencia de factores de riesgo de metástasis linfáticas (Tabla 2).

\section{Tabla 2. Factores de riesgo de metástasis} linfáticas

- Invasión de la submucosa > 500 micras*

- Invasión vascular

- Invasión linfática

* Medido desde la muscular de la mucosa en el punto de máxima invasión.
Sólo se declararon curativas las resecciones que cumplen los 3 criterios mencionados, es decir, resecciones en bloque, de tipo R0 y sin factores de riesgo de metástasis linfáticas. Por protocolo, los pacientes con resecciones en bloque de tipo R0, pero con uno o más factores de riesgo de metástasis linfáticas, son derivados a gastrectomía total o subtotal (de acuerdo a la ubicación del tumor) con disección linfática. La misma conducta se sigue con los pacientes con resecciones no $\mathrm{R}^{27}$.

\section{Seguimiento de los pacientes}

Todos los pacientes son controlados con endoscopia un mes post-procedimiento para evaluar la cicatrización del lecho de resección. Posteriormente se evalúan con endoscopia y biopsias a los 3, 6 y 12 meses, manteniendo seguimiento anual con endoscopia y tomografía computada si no hay nuevos hallazgos.

\section{Resultados}

Entre diciembre de 2011 y junio de 2014, 25 pacientes con diagnóstico de CGI fueron derivados para tratamiento quirúrgico en nuestro Hospital. De estos, 16 casos fueron tratados mediante DSE debido a que cumplían con los criterios de selección.

La mediana de edad de los pacientes tratados con DSE fue 72 años (rango: 61-84). En todos los casos se logró la resección en bloque del tumor. La mediana de tiempo operatorio fue 135 min (rango: 50-320), la mediana de tamaño de las piezas resecadas fue $3,5 \mathrm{~cm}$ (rango: $3-10$ ) y la del tamaño tumoral fue 1,5 cm (rango: 0,7-4,2) (Tabla 3). Hubo una sola complicación que correspondió a una perforación gástrica intra-procedimiento que se trató mediante sutura laparoscópica. La mediana de tiempo de hospitalización fue de 3 días (rango: 2-5). No hubo mortalidad en nuestra serie.

El estudio histológico demostró adenocarcinoma bien diferenciado en 15 pacientes y mixto en 1 (componente bien diferenciado periférico y poco diferenciado central). En 14 pacientes el tumor comprometía exclusivamente la mucosa (T1a) y en 2 pacientes la submucosa (T1b). Todas las resecciones fueron $\mathrm{R} 0$, es decir, con márgenes laterales y profundos libres de tumor. En 14 pacientes la resección fue declarada curativa (Tabla 4). Hubo 2 pacientes en los que a pesar de 
Tabla 3. Características clínicas y resultados perioperatorios de pacientes sometidos a DSE
- Características
- Número de pacientes
- Hombres
8
Edad (años)*
72 (R: 61-84)
- ASA
$-1 \quad 6$
- II
- Perioperatorio
- Tiempo operatorio (minutos)* 135 (R: 50-320)
- Tamaño pieza resecada $(\mathrm{cm})^{*} \quad 3,5$ (R: 3-10)
- Tiempo hospitalización (días)* $3 \quad$ (R: 2-5)

*Datos expresados en mediana con su respectivo rango (R). DSE: Disección submucosa endoscópica. ASA: American Society of Anesthesiologists Classification. haberse realizado una resección en bloque y $\mathrm{R} 0$, no se declaró la resección como curativa. Estos casos correspondieron a un paciente con invasión de la submucosa mayor a 500 micras (SM2) y a un paciente con un adenocarcinoma mixto de 22 mm (Figura 3). En ambos casos se recomendó a los pacientes tratamiento adicional según protocolo consistente en gastrectomía más disección linfática. La biopsia de la pieza operatoria de estos pacientes no demostró metástasis en los ganglios linfáticos. Sin embargo, en el segundo caso, se encontró un segundo tumor, tipo IIb de $10 \mathrm{~mm}$, intramucoso, sin úlcera ni invasión linfovascular, que se encontraba lejos del borde de sección del estómago.

La mediana de seguimiento de la serie es de 15 meses (rango: 2-30). Sólo un paciente ha presentado recidiva de su enfermedad, a los 12 meses de seguimiento, que fue tratada mediante gastrectomía total con disección linfática laparoscópica. La biopsia en este caso demostró un CGI de $7 \mathrm{~mm}$ bien diferenciado, sin metástasis linfáticas.

Tabla 4. Análisis histopatológico de los pacientes sometidos a DSE

\begin{tabular}{|c|c|c|c|c|c|c|c|c|c|}
\hline Caso & $\begin{array}{l}\text { Tipo } \\
\text { tumor }\end{array}$ & $\begin{array}{l}\text { DM Pieza } \\
(\mathbf{c m})\end{array}$ & $\begin{array}{l}\text { DM Tumor } \\
\text { (cm) }\end{array}$ & $\begin{array}{c}\text { Grado } \\
\text { diferenciación }\end{array}$ & SM & $\mathbf{T}$ & $\mathbf{L V}$ & RO & Curativo \\
\hline 1 & Is & 3 & 1,5 & Bien & $(-)$ & T1a & $(-)$ & Si & Si \\
\hline 2 & IIC & 3,5 & 1,2 & Bien & $(-)$ & T1a & $(-)$ & $\mathrm{Si}$ & $\mathrm{Si}$ \\
\hline 3 & $\mathrm{Ilb}$ & 3 & 0,9 & Bien & $(-)$ & T1a & $(-)$ & $\mathrm{Si}$ & $\mathrm{Si}$ \\
\hline 4 & $\|\mathrm{la}+\| \mathrm{c}$ & 4 & 1,5 & Bien & $>500 \mu \mathrm{m}$ & $\mathrm{T} 1 \mathrm{~b}$ & $(-)$ & $\mathrm{Si}$ & No \\
\hline 5 & IIC & 3,5 & 2,2 & Bien & $500 \mu \mathrm{m}$ & $\mathrm{T} 1 \mathrm{~b}$ & $(-)$ & $\mathrm{Si}$ & $\mathrm{Si}$ \\
\hline 6 & IIb & 5 & 3 & Bien & $(-)$ & T1a & $(-)$ & Si & Si \\
\hline 7 & $\|b+\| c$ & 10 & 4,2 & Bien & $(-)$ & T1a & $(-)$ & $\mathrm{Si}$ & $\mathrm{Si}$ \\
\hline 8 & $\|\mathrm{la}+\| \mathrm{c}$ & 3,5 & 1,5 & Bien & $(-)$ & $\mathrm{T} 1 \mathrm{a}$ & $(-)$ & $\mathrm{Si}$ & $\mathrm{Si}$ \\
\hline 9 & $\|b+\| c$ & 5 & 2,2 & Mixto & $(-)$ & $\mathrm{T} 1 \mathrm{a}$ & $(-)$ & $\mathrm{Si}$ & No \\
\hline 10 & IIC & 3,5 & 0,7 & Bien & $(-)$ & T1a & $(-)$ & Si & $\mathrm{Si}$ \\
\hline 11 & IIC & 2,5 & 1 & Bien & $(-)$ & $\mathrm{T1a}$ & $(-)$ & $\mathrm{Si}$ & $\mathrm{Si}$ \\
\hline 12 & IIC & 3,5 & 2 & Bien & $(-)$ & T1a & $(-)$ & $\mathrm{Si}$ & $\mathrm{Si}$ \\
\hline 13 & $\|\mathrm{la}+\| \mathrm{c}$ & 7 & 4 & Bien & $(-)$ & $\mathrm{T} 1 \mathrm{a}$ & $(-)$ & $\mathrm{Si}$ & $\mathrm{Si}$ \\
\hline 14 & IIC & 4 & 2,5 & Bien & $(-)$ & T1a & $(-)$ & $\mathrm{Si}$ & $\mathrm{Si}$ \\
\hline 15 & $\|a+\| c$ & 3 & 1,5 & Bien & $(-)$ & T1a & $(-)$ & $\mathrm{Si}$ & $\mathrm{Si}$ \\
\hline 16 & $\|a+\| c$ & 3,5 & 1,5 & Bien & $(-)$ & $\mathrm{T} 1 \mathrm{a}$ & $(-)$ & $\mathrm{Si}$ & $\mathrm{Si}$ \\
\hline
\end{tabular}

*DSE: Disección endoscópica submucosa, DM: Diámetro mayor, SM: invasión de la submucosa, T: invasión de la pared según la clasificación TNM para cáncer gástrico (AJCC 7ma edición 2010) LV: permeaciones linfovasculares. ${ }^{\S}$ Clasificación Japonesa. 


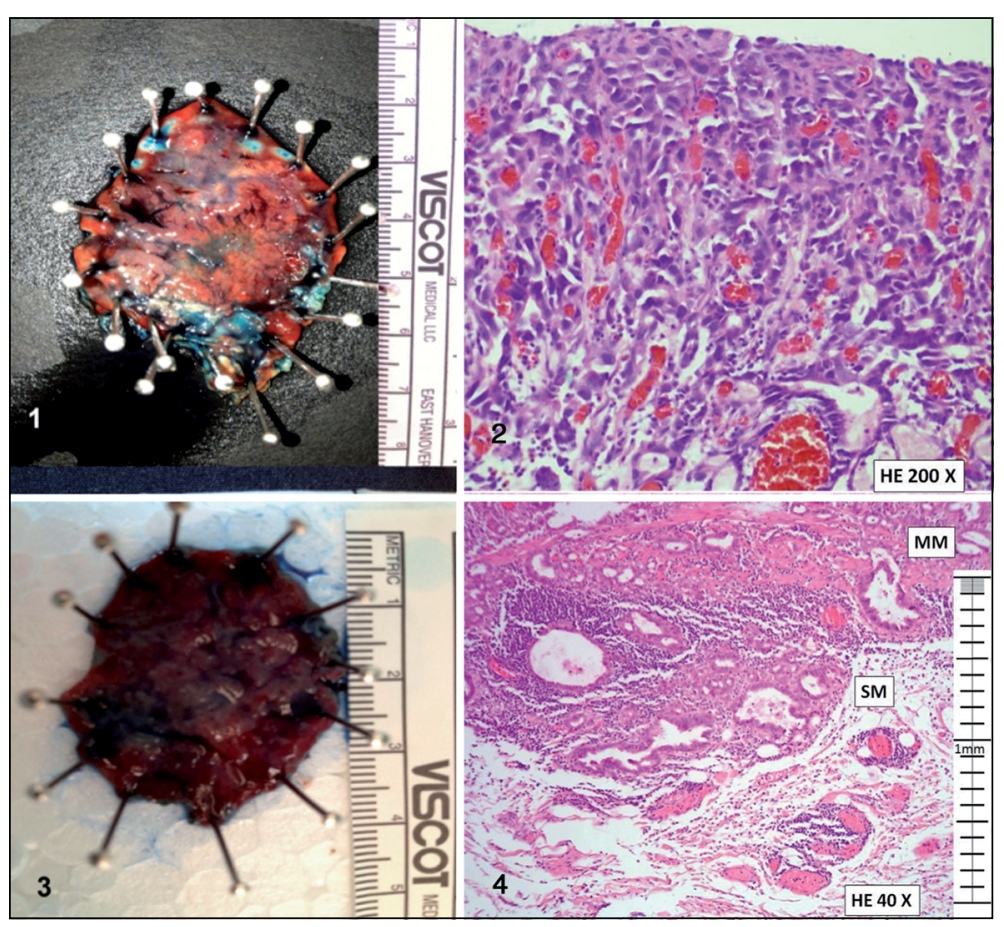

Figura 3. Resecciones no curativas: (1) Lesión Ilb + Ilc, la pieza resecada fue de $5 \mathrm{~cm}$ de diámetro mayor y el tamaño tumoral fue de $2,2 \mathrm{~cm}$ (resección en bloque, R0); (2) La biopsia muestra un adenocarcinoma tubular de tipo mixto, con un componente central mal diferenciado, de tipo intramucoso, no ulcerado, sin permeaciones vasculares ni linfáticas, con bordes negativos; (3) Lesión lla + Ilc, la pieza resecada fue de $4 \mathrm{~cm}$ de diámetro mayor y el tamaño tumoral fue de $1,5 \mathrm{~cm}$ (resección en bloque, R0); (4) La biopsia muestra un adenocarcinoma tubular bien diferenciado con invasión de la submucosa mayor a $500 \mu \mathrm{m}$ (SM3). Ambas resecciones fueron declaradas no curativas, y se realizó gastrectomía total laparoscópica con disección linfática en ambos casos. (Mm: muscular de la mucosa, Sm: submucosa).

\section{Discusión}

El presente trabajo describe nuestra experiencia inicial y protocolo de DSE en CGI. Si bien el seguimiento es aún a corto plazo, creemos que este trabajo tiene el valor de presentar un protocolo ordenado y estricto en cuanto a la selección de pacientes, a su seguimiento y con una importante proyección a futuro en una técnica que ha tenido hasta el momento escaso desarrollo en occidente. Otro aspecto importante a destacar es el trabajo en conjunto con el equipo de Anatomía Patológica. Al respecto debemos recordar que los pacientes con CGI son justamente los que tienen la mayor probabilidad de curación, por lo que el análisis de la pieza operatoria debe ser estricto y protocolizado, de manera de poder declarar con seguridad como curativos o no curativos los procedimientos y de esta manera poder determinar si requerirán o no algún tratamiento adicional.

En esta experiencia todas las resecciones se completaron en forma exitosa, con márgenes macroscópicos y microscópicos libres de tumor. A pesar de esto, en 2 pacientes la resección fue calificada como no curativa debido a la existencia de factores de riesgo de metástasis linfáticas en el análisis final de la pieza operatoria. En ambos casos, la probabilidad de presentar compromiso linfático era baja, probablemente cercana a $6 \%{ }^{17}$. Considerando que este porcentaje supera la mortalidad asociada a una gastrectomía ${ }^{28}$ se recomendó cirugía a los 2 pacientes. Si bien la biopsia final no demostró metástasis linfáticas en ninguno de los casos, creemos que esta es la conducta que se debe tener hasta no contar con mayor evidencia al respecto, teniendo siempre como primer objetivo la seguridad oncológica de nuestros pacientes.

Es importante destacar el hallazgo post-DSE de un adenocarcinoma mixto. En este caso, la biopsia pre-DSE informó sólo adenocarcinoma bien diferenciado, (motivo por el que fue seleccionado para DSE). Respecto a esto, el pronóstico de los adenocarcinomas mixtos y su comportamiento biológico aún no se ha esclarecido completamente ${ }^{29,30}$. Existen trabajos que sugieren que los adenocarcinomas mixtos podrían tener aún peor pronóstico que los adenocarcinomas poco diferenciados y un mayor riesgo de recurrencia post-DSE ${ }^{31,32}$. Esto junto al tamaño tumoral (mayor a $20 \mathrm{~mm}$ ) fue considerado para recomendar tratamiento quirúrgico adicional al paciente.

El hallazgo de un tumor sincrónico post-gas- 
trectomía en este mismo caso es un hecho que merece consideración. Si bien las lesiones sincrónicas se han descrito hasta en $14 \%$ de los casos de $\mathrm{CGI}^{33}$, el no haber detectado la lesión en la endoscopia pre-DSE es un elemento que podría generar preocupación respecto al carácter oncológico de una terapia local como la DSE. Por otra parte, es importante destacar que esta lesión correspondía a un tumor tipo IIb. Este tipo de lesiones son ciertamente las de más difícil diagnóstico, pudiendo pasar desapercibidas incluso si la endoscopia es realizada en un centro de alta experiencia ${ }^{34,35}$. Más aún, este riesgo no es diferente al de los pacientes sometidos a gastrectomía sub-total, en los que también podrían pasar desapercibidas este tipo de lesiones en el estómago proximal no resecado. Este hecho plantea un importante desafío, no sólo en el diagnóstico, sino también en el estricto seguimiento al que deben ser sometidos todos los pacientes tratados por cáncer gástrico, ya sea mediante DSE o por gastrectomía.

Otro punto importante a discutir son las complicaciones asociadas a la DSE. Tanto la hemorragia como la perforación gástrica se han descrito hasta en $7 \%$ y $4 \%$ respectivamente ${ }^{36}$. En esta serie no hubo casos de hemorragia. Sin embargo, se produjo una perforación que fue tratada mediante sutura laparoscópica. Este caso correspondió a la lesión más grande de la serie (pieza de resección de $10 \mathrm{~cm}$ ) y el paciente evolucionó favorablemente. Si bien nuestro número de pacientes es aún escaso para obtener porcentajes y comparar cifras, es esperable que al comienzo podamos tener una mayor incidencia de complicaciones comparado a los grupos asiáticos que cuentan con mayor experiencia y una gran casuística.

En Japón, la mayoría de las perforaciones en DSE son tratadas con clips por vía endoscópica ${ }^{37}$. En nuestro grupo acordamos por protocolo tratar esta complicación mediante sutura laparoscópica durante la experiencia inicial. Es probable que en el futuro tratemos este tipo de complicaciones también por vía endoscópica.

Todos los pacientes de esta serie se encuentran en estricto seguimiento de acuerdo a protocolo y hasta el momento se ha detectado sólo una recidiva. Este caso corresponde a una mujer de 74 años con un tumor submucoso SM1 límite (invasión de la submucosa de 500 micras). La recidiva fue detectada a los 12 meses post-DSE y la biopsia de la gastrectomía demostró un CGI bien diferenciado sin metástasis linfáticas, por lo que la paciente mantiene un excelente pronóstico y una alta probabilidad de curación. Este caso resalta la importancia del trabajo multidisciplinario, protocolizado y de un estricto seguimiento.

Creemos que en casos debidamente seleccionados, la DSE presenta importantes ventajas para los pacientes. En nuestra serie 4 casos correspondían a tumores sub-cardiales, 6 casos a tumores corporales y 6 casos a tumores ubicados en el antro. Si estos pacientes se hubiesen operado, 4 se hubiesen tratado mediante gastrectomía total y 12 hubiesen requerido una gastrectomía sub-total. Ambas cirugías no están exentas de morbilidad ni tampoco de mortalidad ${ }^{25}$, y si bien hasta nuestro conocimiento no hay estudios comparativos de calidad de vida entre gastrectomía y DSE, creemos que el hecho de preservar el estómago presenta importantes ventajas en cuanto a calidad de vida para nuestros pacientes. En ellos, el seguimiento endoscópico periódico es indispensable.

Por último, se debe enfatizar la importancia del diagnóstico precoz del cáncer gástrico, y a este objetivo deben apuntar los estándares actuales del diagnóstico endoscópico. Junto a la implementación de esta técnica, nuestro grupo al igual que muchos otros centros en Chile ${ }^{38,39}$ ha trabajado arduamente durante el último tiempo en aumentar la detección de lesiones incipientes. Es de esperar que estos esfuerzos permitan en el futuro aumentar el bajo porcentaje de CGI comunicado históricamente en las series nacionales, ya que esto ciertamente podría tener un impacto positivo en la mortalidad por cáncer gástrico en Chile.

En resumen, el presente trabajo describe nuestra primera experiencia en DSE en CGI, demostrando la factibilidad de implementar este procedimiento en forma segura, protocolizada, y con buenos resultados inmediatos y a corto plazo. Los resultados de esta técnica deberán ser estrictamente evaluados en un seguimiento a largo plazo y con un mayor número de pacientes.

\section{Referencias}

1. Instituto Nacional de Estadísticas. Anuario de Estadísticas Vitales 2010. http://www.ine.cl/canales/chile_estadistico/demografia_y_vitales/estadisticas_vitales/pdf/ vitales_2010.pdf

2. Serra I, Báez S, Serra J, Calvo A, Decinti E. Evolución 
epidemiológica reciente del cáncer gástrico en Chile y el mundo. Rev Chil Cir 1997; 129: 749-55.

3. Llanos O, Butte JM, Crovari F, Duarte I, Guzmán S. Survival of young patients after gastrectomy for Gastric Cancer. World J Surg 2006: 30; 17-20.

4. Burmeister R, Pepper J, Fernández M. Sobrevida a largo plazo del cáncer gástrico. Rev Chil Cir 1986; 38: 193-96.

5. Bozzetti F, Marubini E, Bonfanti G, Miceli R, Piano C, Crose N, et al. Total versus subtotal gastrectomy: surgical morbidity and mortality rates in a multicenter Italian randomized trial. The Italian Gastrointestinal Tumor Study Group. Ann Surg 1997; 226 (5): 613-20.

6. Bartlett EK, Roses RE, Kelz RR, Drebin JA, Fraker DL, Karakousis GC. Morbidity and mortality after total gastrectomy for gastric malignancy using the American College of Surgeons National Surgical Quality Improvement Program database. Surgery 2014; 156 (2): 298-304.

7. Tyrväinen T, Sand J, Sintonen H, Nordback I. Quality of life in the long-term survivors after total gastrectomy for gastric carcinoma. J Surg Oncol 2008; 97: 121-4.

8. Seung SL, Ho YCh, Wansik Y. Quality of Life of LongTerm Survivors after a Distal Subtotal Gastrectomy. Cancer Res Treat 2010; 42 (3): 130-4.

9. Japanese Gastric Cancer Association. Japanese classification of gastric carcinoma-2nd English edition. Gastric Cancer 1998; 1: 10-24.

10. Everett SM, Axon ATR. Early gastric cancer in Europe. Gut 1997; 41: 142-50.

11. Butte JM, Torres J, Viviani P, Duarte I, Crovari F, Guzmán $\mathrm{S}$, et al. Sobrevida alejada de pacientes operados por cáncer gástrico incipiente. Rev Med Chile 2008; 136: 1424-30.

12. Ceroni M, García C, Benavides C, Covacevich S, Rubilar $\mathrm{P}$, Cid et al. Seguimiento mayor a 10 años de pacientes operados por cáncer gástrico incipiente. Rev Chil Cir 2011; 63: 591-8.

13. Gotoda T, Yamamoto H, Soetikno RM. Endoscopic submucosal dissection of early gastric cancer. J Gastroenterol 2006; 41: 929-42.

14. Oda I, Saito D, Tada M. A multicenter study of endoscopic resection for early gastric cancer. Gastric Cancer 2006; 9: 262-70.

15. Gotoda T, Iwasaki M, Kusano C, Seewald S, Oda I. Endoscopic resection of early gastric cancer treated by guideline and expanded National Cancer Center criteria. Br J Surg 2010; 97: 868-71.

16. Japanese Gastric Cancer Association. Japanese gastric cancer treatment guidelines 2010 (ver. 3). Gastric Cancer 2011; 14: 113-23.

17. Gotoda T, Yanagisawa A, Sasako M, Ono H, Nakanishi $\mathrm{Y}$, Shimoda $\mathrm{T}$, et al. Incidence of lymph node metastasis from early gastric cancer: estimation with a large number of cases at two large centers. Gastric Cancer 2000; 3 : 219-25.

18. Osawa H, Yamamoto H, Miura Y, Yoshizawa M, Sunada $\mathrm{K}$, Satoh K, et al. Diagnosis of extent of early gastric cancer using flexible spectral imaging color enhancement. World J Gastrointest Endosc 2012; 4 (8): 356-61.

19. Yao K, Anagnostopoulos GK, Ragunath K. Magnifying endoscopy for diagnosing and delineating early gastric cancer. Endoscopy 2009; 41: 1-6.

20. Kwee RM, Kwee TC. The accuracy of endoscopic ultrasonography in differentiating mucosal from deeper gastric cancer. Am J Gastroenterol 2008; 103 (7): 1801-9. Epub 2008 Jun 28.

21. Donoso A, Sharp A, Gellona J, Parra-Blanco A, Pimentel F, Escalona A, et al. Disección sub-mucosa endoscópica en cáncer gástrico incipiente. Rev Chil Cir 2013; 65 (2): 180-6.

22. Toyonaga T, Inokuchi H, Man-I M, Morita Y, Yoshida M, Kutsumi H, et al. Endoscopic submucosal dissection using water jet short needle knives (Flush knife) for the treatment of gastrointestinal epitelial neoplasm. Acta Endoscopica 2007; 37: 646-56.

23. Conlin A, Kaltenbach T, Kusano C, Matsuda T, Oda I, Gotoda T. Endoscopic resection of gastrointestinal lesions: advancement in the application of endoscopic submucosal dissection. J Gastroenterol Hepatol 2010; 25 (8): 1348-57.

24. Ohkuwa M, Hosokawa K, Boku N, Ohtu A, Tajiri H, Yoshida S. New Endoscopic Treatment for Intramucosal Gastric Tumors Using an Insulated-Tip Diathermic Knife. Endoscopy 2001; 33 (3): 221-6.

25. Parra-Blanco A, Uraoka T, Ortiz J, Fernández S, Alonso $\mathrm{H}$, Amor $\mathrm{P}$, et al. ¿Es útil un método de tracción (clip-banda) para facilitar la disección submucosa gástrica?. Estudio prospectivo, aleatorizado, controlado en modelo porcino in-vivo. Endoscopy 2011; 43 A9.

26. Toyonaga T, Man-i M, East J, Nishino E, Ono W, Hirooka T, et al. 1635 Endoscopic submucosal dissection cases in esophagus, stomach and colorectum: complication rates and long-term outcomes. Surg Endosc 2013; 27: $1000-8$.

27. Oda I, Gotoda T, Sasako M, Sano T, Katai H, Fukagawa $\mathrm{T}$, et al. Treatment strategy after non-curative endoscopic resection of early gastric cancer. British Journal of Surgery 2008; 95: 1495-500.

28. Papenfuss WA, Kukar M, Oxenberg J, Attwood K, Nurkin S, Malhotra U, et al. Morbidity and Mortality Associated with Gastrectomy for Gastric Cancer. Ann Surg Oncol 2014. Apr 4. (Epub ahead of print).

29. Iwamoto J, Mizokami Y, Ito M, Shomokobe K, Hira- 
yama T, Honda A, et al. Clinicopathological features of undifferentiated mixed type early gastric cancer treated with endoscopic submucosal dissection. Hepatogastroenterology 2010; 57: 185-90.

30. Nakata K, Nagai E, Miyasaka Y, Ohuchida K, Ohtsuka $\mathrm{T}$, Toma $\mathrm{H}$, et al. The risk of lymph node metastases in mucosal gastric carcinoma: especially for a mixture of differentiated and undifferentiated adenocarcinoma. Hepatogastroenterology 2012; 59: 1855-8.

31. Han JP, Hong SJ, Kim HK. Long term outcomes of early gastric cancer diagnosed as mixed adenocarcinoma after endoscopic submucosal dissection. Journal of Gastroenterology and Hepatology 2015; 30: 316-20.

32. Komatsu Sh, Ichikawa D, Miyamae M, Shimizu H, Konishi H, Shiozaki A, et al. Histological mixed-type as an independent prognostic factor in stage I gastric carcinoma. World J Gastroenterol 2015; 21(2): 549-55.

33. Lee IS, Park YS, Kim KC, Kim TH, Kim HS, Choi KD, et al. Multiple Synchronous early gastric cancers: High-risk group and proper management. Surgical Oncology 2012; 21: 269-73.

34. Kim HH, Cho EJ, Noh E, Choi SR, Park SJ, Park MI, et al. Missed synchronous gastric neoplasm with endoscopic submucosal dissection for gastric neoplasm: experience in our hospital. Digestive Endoscopy 2013;
25 (1): 32-8.

35. Jang MY, Cho JW, Oh WG, Ko SJ, Han SH, Baek HK, et al. Clinicopathological characteristics of synchronous and metachronous gastric neoplasm after endoscopic submucosal dissection. Korean J Intern Med 2013; 28 (6): 687-93.

36. Oda I, Gotoda T, Hamanaka H, Eguchi T, Saito Y, Matsuda T, et al. Endoscopic submucosal dissection for early gastric cancer: technical feasibility, operation time and complications from a large consecutive cases. Dig Endosc 2005; 17: 54-8.

37. Minami S, Gotoda T, Ono H, Oda I, Hamanaka H. Complete endoscopic closure using endoclips for gastric perforation during endoscopic resection for early gastric cancer can avoid emergent surgery. Gastrointest Endosc 2006; 63: 596-601.

38. Calvo A, Pruyas M, Nilsen E, Verdugo P. Pesquisa poblacional de cáncer gástrico en pacientes sintomáticos digestivos, período 1996-2000. Rev Med Chile 2001; 129 (7): 749-55.

39. Rollán A, Cortés P, Calvo A, Araya R, Bufadel M, González R, et al. Diagnóstico precoz de cáncer gástrico. Propuesta de detección y seguimiento de lesiones premalignas gástricas: protocolo ACHED. Rev Med Chile 2014; 142: 1181-92. 